\title{
Strangulated small bowel through vaginal vault rupture: late complication of abdominal sacrocolpopexy
}

\author{
O. Eskandar $\cdot$ J. Hodge $\cdot$ S. Eckford
}

Received: 18 August 2008 / Accepted: 6 October 2008 / Published online: 4 November 2008

(C) Springer-Verlag 2008

\begin{abstract}
Sacrocolpopexy is currently a favourable procedure for management of apical defect and vaginal vault prolapse. Recently, it has been extensively evaluated in terms of its efficacy, durability and potential short- and long-term complications. These complications have been investigated by many authors, including urinary retention, urge incontinence, urinary tract infections, wound infection, haematomas, bowel symptoms and gastrointestinal complications. However, we report the first case of strangulated small bowel due to herniation through vaginal vault rupture as a late complication of sacrocolpopexy. This report reviews the risk factors and precipitating causes of bowel evisceration particularly after sacrocolpopexy, and peri- and intraoperative preventive measures are discussed, as well as various management modalities.
\end{abstract}

Keywords Sacrocolpopexy · Vault rupture - Evisceration . Bowel herniation $\cdot$ Strangulation $\cdot$ Mesh erosion

\section{Introduction}

Abdominal sacrocolpopexy remains the preferred option over vaginal sacrospinous fixation for post-hysterectomy

\footnotetext{
O. Eskandar $(\bowtie) \cdot J$. Hodge $\cdot$ S. Eckford

Department of Obstetrics and Gynaecology,

North Devon District Hospital,

Barnstaple,

Devon EX31 4JB, UK

e-mail: oeskandar@yahoo.com

J. Hodge

e-mail: jack.hodge@ndevon.swest.nhs.uk

S. Eckford

e-mail: seumas.eckford@ndevon.swest.nhs.uk
}

vault prolapse in terms of reduced recurrent prolapse rates. Complications of abdominal sacrocolpopexy are common but usually self-limiting (urinary retention, urge incontinence, urinary tract infections, wound infections, haematomas, etc). Mesh erosion is a significant concern, especially when the mesh is placed vaginally. Serious complications are very uncommon.

We report here the first case of strangulated small bowel due to herniation through vaginal vault rupture as a late complication of sacrocolpopexy.

\section{Case report}

A 75-year-old woman was referred as an emergency from primary care. She found "something" prolapsing from her vagina after she had blown her nose and felt something "give way" inside. She presented $6 \mathrm{~h}$ later, with lower abdominal pain and vomiting. She was hypertensive and hypothyroid and was undergoing a course of palliative chemotherapy (Epirubicin, cisplatin, 5-FU) for an inoperable oesophageal cancer, which had been diagnosed 7 months earlier. She had been celibate for several years.

She had undergone vaginal hysterectomy and pelvic floor repair 22 years previously for uterovaginal prolapse. She had undergone a posterior vaginal repair and anterior vaginal repair with Surgisis ${ }^{\circledR}$ Soft Tissue Graft (Cook Biotech Incorporated (CBI), West Lafayette, IN, USA) 4 years previously. Three years previously, she had undergone open abdominal sacrocolpopexy for vaginal vault prolapse and recurrent cystocele. Type I macroporous $(>70 \mu \mathrm{m})$ polypropylene mesh (Ethicon Endo-Surgery, Johnson and Johnson, Cincinnati, OH, USA) was sutured by monofilament, non-braided permanent suture (Prolene size 0 ) to the vaginal vault and with titanium spiral staples 


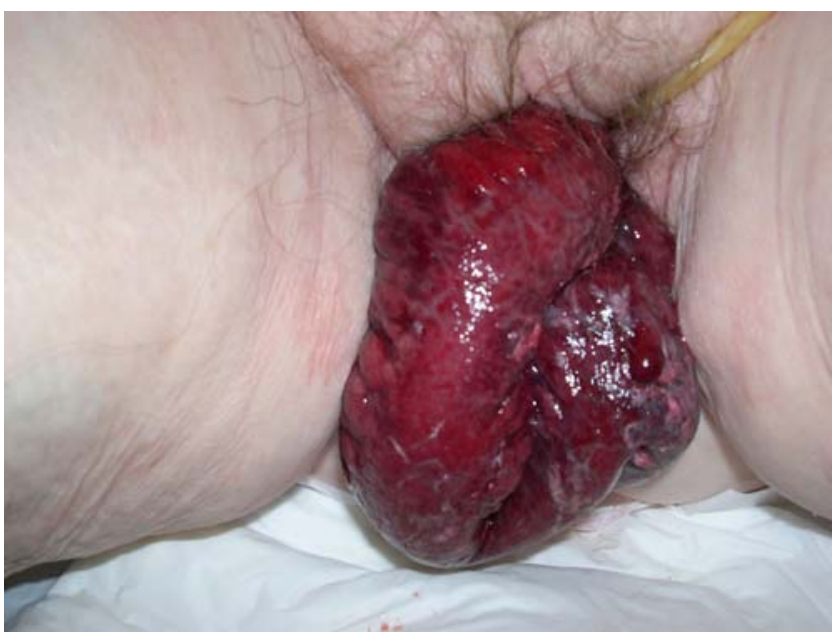

Fig. 1 A loop of small intestine prolapsed from the vagina shows marked congestion, bleeding and beginning of ulceration and gangrene

(Protack $5 \mathrm{~mm}$ autosuture, Tyco Healthcare stapler) to the pre-sacral longitudinal ligaments approximately at the level of the third sacral segment (S-3). The mesh was reperitonealised. Prophylactic antibiotics were given with anaesthetic induction. At 6 weeks post-operative review, pelvic examination revealed a well-healed and supported vaginal vault with no granulation tissues or evidence of mesh erosion.

On current examination, she was haemodynamically stable; the abdomen was soft with no signs of peritonitis. There was an obvious herniation of the small bowel through the vagina. The bowel was markedly congested, and there were early mucosal ulcerations and ischaemic changes and mucosal necrosis (Fig. 1). All haematological and biochemical blood tests were within normal limits, including full blood count and urea and electrolytes. Speculum examination was impossible because the markedly oedematous prolapsed bowel obscured the vaginal introitus. A $2-\mathrm{cm}$ defect was felt at the vaginal vault through which a loop of the small intestine was prolapsing. The hole was tightly strangulating the bowel at the apex of the vagina. A trial of external reduction of the herniated bowel was unsuccessful. The prolapsed bowel was wrapped with warm moist saline towels, and a course of intravenous Co-amoxiclav was commenced. Two hours later, an emergency laparotomy was performed though a midline exploratory abdominal incision; the prolapsed small bowel was reduced, with difficulty, by a combined vaginal and abdominal approach. After bowel reduction, the prolapsed piece of small intestine still appeared non-viable and severely congested with areas of ulcerations and gangrene. A resection of the non-viable loop of the ileum was performed with re-anastomosis of the healthy margins. A remnant of the mesh, used for the sacrocolpopexy, was found loosely attached to the vault of the vagina, which was dissected and removed. The vaginal tear was closed with interrupted braided synthetic absorbable polyglactin Vicryl No (1) suture. The post-operative period was unremarkable, and she was discharged from the hospital a week later. At 6-week follow-up, the patient was asymptomatic, and the abdominal wound was found well healed. The histopathological examination of the excised section of small intestine showed a $47-\mathrm{cm}$ length of ileum with extensive serositis. Both end resection margins were viable. Vaginal examination revealed a well-healed vaginal vault with a moderate degree of vault prolapse and cystocele; however, the patient did not wish any further surgical intervention at that stage.

\section{Discussion}

Vaginal rupture and bowel evisceration is an extremely rare life-threatening clinical condition that requires immediate surgical management. A search of Medline from January 1950 to April 2008 was carried out in a non-restricted language, with a key word search using "sacrocolpopexy", "vault rupture", "vaginal rupture", "evisceration", "bowel herniation", "strangulation" and "mesh erosion".

There have been very few cases, in the literature, since McGregor in 1907 reported the first case of spontaneous vaginal rupture as cited [1]. It is relatively more common in post-menopausal women, presumably due to the hypoestrogenism and consequent vaginal atrophy, thinning and de-vascularisation of previously healthy vaginal tissue. A short atrophic vagina is a particular risk for rupture [2]. However, in pre-menopausal women, vaginal rupture is less common and usually follows vaginal trauma by vigorous sexual intercourse, introduction of vaginal foreign bodies and traumatic vaginal deliveries.

Hysterectomy, either abdominal and more commonly vaginally, and previous vaginal prolapse repair particularly enterocele and are recognised as risk factors for vaginal rupture. Dissection during vaginal surgery devascularises tissues and compromises the integrity of vaginal fascia and encourages vaginal shortening [1]. Prolonged use of vaginal pessaries for vaginal prolapse may lead to stretching, thinning, ulceration and weakness of the vaginal tissues and increase the risk of vaginal rupture particularly if vaginal surgical repair is subsequently used to manage the prolapse.

Other risk factors for vaginal rupture include connective tissue disorders, pelvic irradiation, post-operative vault haematomas and infection, corticosteroid therapy, high body mass index and smoking. Another possible risk factor specific to this present case is the fact that the patient had a 
course of chemotherapy for oesophageal cancer. It has been reported that chemotherapeutic agents, especially anthracyclines (e.g. aclarubicin, daunorubicin, doxorubicin, [3] epirubicin and idarubicin) predispose to mucositis and vaginal atrophy.

Vaginal rupture usually follows a direct precipitating cause due to sudden rise in the intra-abdominal pressure such as straining during defection, coughing, blowing the nose or heavy lifting.

Sacrocolpopexy is a particular risk for vaginal rupture because it is usually performed in patients who have vaginal vault prolapse often with several previous pelvic surgeries, commonly in post-menopausal patients, and usually, it is associated with enterocele. Insertion of synthetic mesh in sacrocolpopexy may lead to further weakness of the vaginal fascia particularly if it is complicated with infection and mesh erosion. Furthermore, sacrocolpopexy changes the normal axis of the vagina as it brings the vagina in a more vertical plan rather than its normal anatomical almost horizontal plan resting on the levator plate. The angle of inclination of the upper vagina to the horizontal is normally less than $45^{\circ}$ [4]. During increased intra-abdominal pressure, the vaginal axis becomes even more horizontal, and the angle of inclination is reduced to virtually zero so that the vagina is maintained well supported above the levator plate [5]. This important mechanism protects the vaginal vault, which is the weakest part of the vagina, during straining. Moreover, the tension created by the mesh of the sacrocolpopexy on the vaginal apex adds to the weakness of the vaginal vault and increases the risk of tearing and rupture of the vagina.

Post-hysterectomy vaginal shortening is a risk factor for vaginal rupture in patients subsequently undergoing sacrocolpopexy, as the shortened vagina lies directly in the plane of the genital hiatus unsupported by the levator plate and prolapses more readily, making it more prone to rupture. Further, short vagina requires a relatively greater length of mesh. This may increase the risk of vaginal stretching and avulsion of the mesh from the vault secondary to tension from the mesh as it shrinks and retracts. It has been shown that polypropylene meshes shrink to about $30-50 \%$ of their original size after 4 weeks of insertion in animal models [6] and also when removed after abdominal hernia repair [7].

The risk of vaginal rupture following sacrocolpopexy can be reduced by adapting the surgical technique to reduce the tension on the mesh attached to the vaginal apex with a tendency of under-correction of vault prolapse that makes the vagina less stretched and leaving the vagina more horizontal rather than vertical to reduce risk of vaginal rupture. Furthermore, deep fixation of the mesh, preferably at S-3 segment, may reduce the risk of vault rupture as this makes vaginal axis more horizontal. Furthermore, the use of minimal amount of mesh accompanied with tension-free application with avoidance of combined abdominal and vaginal surgery minimise the potential risk of complication.

Peri-operative prophylactic antibiotic reduces infection, and mesh erosion rates consequently reduce the risk of vaginal rupture. Coexistent vaginal atrophy should be treated with hormonal therapy to minimise risk of vaginal tearing during apical suturing. Pre-operative assessment is essential to identify high-risk patients with multiple previous prolapse operations with possible atrophic and very thin vaginal vault. Non-sacropexy solutions to apical prolapse, e.g. sacrospinous fixation may be considered for those patients. Nevertheless, vaginal rupture and bowel evisceration have been reported following sacrospinous vaginal vault fixation [8]. Furthermore, randomised trials $[9,10]$ have clearly demonstrated that abdominal techniques are superior to the vaginal approach.

Furthermore, pre-operative counselling is essential, and patients should be advised to avoid post-operative chronic increase in intra-abdominal pressure by avoiding heavy lifting, weight gain, smoking and chronic constipation.

Vaginal rupture and bowel evisceration can be repaired vaginally, abdominally or by a combined approach. Laparoscopic management has been described. Concomitant surgical repair of associated vaginal prolapse and pelvic floor repair during surgical management of evisceration has been advocated by some authors; however, others recommend delayed pelvic repair after the primary tissue rupture sites have healed. Cul-de-sac obliteration or colpocleisis have been suggested in patients who wish to abstain from sexual intercourse [2].

\section{References}

1. Karam JA, Wengert PA, Kerstein MD (1995) Vaginal evisceration. South Med J 88(3):355-356, ISSN: 0038-4348, Mar

2. Croak AJ, Gebhart JB, Klingele CJ, Schroeder G, Lee RA, Podratz KC (2004) Characteristics of patients with vaginal rupture and evisceration. Obstet Gynecol 103(3):572-576, ISSN: 00297844, Mar

3. Krychman ML, Carter J, Aghajanian CA, Dizon DS, Castiel M (2004) Chemotherapy induced dyspareunia: a case study of vaginal mucositis and pegylated liposomal doxorubicin injection in advanced stage ovarian carcinoma. Gynecol Oncol 93(2):561563, ISSN: 0090-8258, May

4. Petros PE (2001) Vault prolapse I: dynamic supports of the vagina. Int Urogynecol J Pelvic Floor Dysfunct 12(5):292-295, ISSN: 0937-3462

5. Virtanen HS, Ekholm E, Kiilholma PJ (1996) Evisceration after enterocele repair: a rare complication of vaginal surgery. Int Urogynecol J Pelvic Floor Dysfunct 7(6):344-347, 27 refs, ISSN: 0937-3462

6. Klinge U, Klosterhalfen B, Müller M, Ottinger AP, Schumpelick V (1998) Shrinking of polypropylene mesh in vivo: an experimental study in dogs. Eur J Surg 164(12):965-969, ISSN: 11024151, Dec

7. Baessler K, Maher-Christopher F (2006) Mesh augmentation during pelvic-floor reconstructive surgery: risks and benefits. Curr 
Opin Obstet Gynecol 18(5):560-566, 51 refs, ISSN: 1040-872X, Oct

8. Farrell SA, Scotti RJ, Ostergard DR, Bent AE (1991) Massive evisceration: a complication following sacrospinous vaginal vault fixation. Obstet Gynecol 78(3 Pt 2):560-562, ISSN: 0029-7844, Sep

9. Benson JT, Lucente V, McClellan E. (1996) Vaginal versus abdominal reconstructive surgery for the treatment of pelvic support defects: a prospective randomized study with long-term outcome evaluation. [Clinical Trial. Comparative Study. Journal Article. Randomized Controlled Trial] Am J Obstet Gynecol 175 (6):1418-21; discussion 1421-2, Dec

10. Maher C, Baessler K, Glazener CM, Adams EJ, Hagen S, (2004) Surgical management of pelvic organ prolapse in women. [update in Cochrane Database Syst Rev. 2007;(3):CD004014; PMID: 17636742]. [Review] [44 refs] [Journal Article. Meta-Analysis. Review] Cochrane Database of Systematic Reviews (4): CD004014 